\title{
Theoretical study of gallium nitride nanocage as a carrier for Cisplatin anticancer drug
}

\author{
Merve Şenğül ALPATER ${ }^{1}$, Zaid H. AL-SAWAFF 2, 3, ${ }^{*}$ and Fatma KANDEMİRLİ 1 \\ ${ }^{1}$ Biomedical Engineering Department, Faculty of Engineering and Architecture, Kastamonu University, Kastamonu, 3700 \\ Turkey. \\ 2 Materials Science and Engineering, Faculty of Engineering and Architecture, Kastamonu University, Kastamonu, 3700 \\ Turkey. \\ ${ }^{3}$ Medical Instrumentation Technology, Technical Engineering College, Northern Technical University, Mosul, Iraq.
}

Global Journal of Engineering and Technology Advances, 2021, 09(03), 077-085

Publication history: Received on 14 November 2021; revised on 19 December 2021; accepted on 21 December 2021

Article DOI: https://doi.org/10.30574/gjeta.2021.9.3.0166

\begin{abstract}
In this paper, the possible interactions between cisplatin Cl2H6N2Pt as an anticancer drug and gallium nitride (Ga12N12) nanocage have been investigated using the DFT/b3lyp/lanl2dz(d,p) level of theory. Three different orientations were used to mimic the cisplatin adsorbed on Ga12N12. To investigate the interaction mechanism between the two components, the adsorption energies and thermodynamic parameters, the electronic properties such as the energies and orbitals distribution of the highest occupied molecular orbital (HOMO), the lowest unoccupied molecular orbital (LUMO), the HOMO-LUMO energy gaps (Eg), thermodynamic properties were also investigated. Additionally, some quantum molecular descriptors were calculated to understand molecular reactivity. The main results revealed that the adsorption process of the drug compound on the surface of the nanocage varies with the adsorption site. The process showed that different energies could be obtained, where the highest energy value was when the drug compound was adsorbed with the nanocage at the chlorine atom, with a value of (41.85) kcal/mol. On the other hand, the distance between the drug compound atoms was affected before and after adsorption, which proves the existence of an interaction between the drug compound and the nanocage and considers it as a drug delivery vehicle.
\end{abstract}

Keywords: Drug delivery; Cisplatin drug; Gallium nitride nanocage; DFT calculations; Thermodynamics

\section{Introduction}

Cisplatin $\mathrm{Cl}_{2} \mathrm{H}_{6} \mathrm{~N}_{2} \mathrm{Pt}$ (Fig1 (b)) is a type of drug used in chemotherapy and cancer treatments. Rapid and inactive cisplatin undergoes enzymatic changes and turns into metabolites. Cisplatin exits the body mainly through urine. If the binding to proteins and tissues in the body is too high, it causes the elimination phase to be prolonged or incomplete. Cisplatin is used in certain types of cancer, either alone or in combination with other approved chemotherapeutic agents [1].

The use of nanotechnology in medicine leads to interesting applications, such as using nanoparticles to deliver drugs to cancer cells, detecting cancer cells in the bloodstream, repairing specific cancer cells, or as an antibacterial. The use of nanoparticles to deliver medicines to cancerized cells allows the drug particles to be engineered to focus on cancerized cells; hence, direct treatment of those cells is achieved. This procedure reduces the side effects and decreases damage to healthy cells in the body [2]. Nanocage (NC) particle is a hollow fullerene-like structure. It is an essential building block for nanotechnology applications because of its larger surface area and the empty central region that allow both exohedral and endohedral functionalization that helps to fine-tune the physical and chemical properties [3]. Nanocages

\footnotetext{
* Corresponding author: Zaid H AL-SAWAFF

Medical Instrumentation Technology, Technical Engineering College, Northern Technical University, Mosul, Iraq. 
have gained research attention because of their excellent properties such as high oxidation, thermal conductivity, temperature stability, and constant bandgap with semiconductor nature [4].

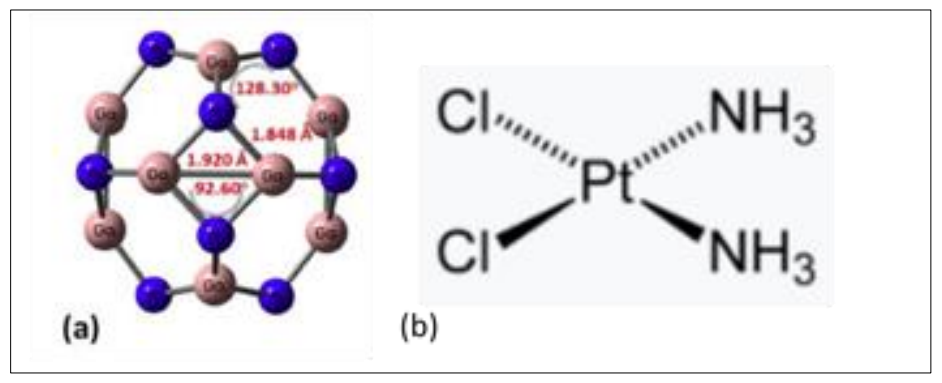

Figure 1 Chemical structure of (a) Ga12N12 nanocage, (b) cisplatin molecule

A group of previous studies on the adsorption of cisplatin on the surfaces of a group of nanoparticles was reported by Badiee $\mathrm{M}$ et al. Using quantum mechanics, the interaction of cisplatin (CPT) with $(5,5)$ single-walled carbon nanotubes (SWNT) was studied. Calculations were performed using the hybrid density functional method (B3LYP) in liquid phases. Three modes of non-covalent and covalent interactions of cisplatin on pristine SWNT were investigated. Quantitative molecular descriptors and frontier orbital analysis have been studied in drug nanotube systems. It was found that the binding of cisplatin to pristine (CPT/NT) in the solution phase is thermodynamically appropriate [5].

On the other hand, first-principles calculations based on DFT were performed to investigate the adsorption behavior of cisplatin (CP) on BN (PBN), Al-doped BN (Al-BN), and Ga doped BN (Ga-BN) nanosheets in gas and water media by Piya A et al.. They reported the calculated adsorption energy of the CP/PBN complex is about $-0.87 \mathrm{eV}$ and $-0.41 \mathrm{eV}$ in the gas and water phases, respectively. But after doping $\mathrm{Al}$ and $\mathrm{Ga}$ atom on the $\mathrm{BN}$ nanosheet separately, the adsorption energies were greatly improved to $-2.12 \mathrm{eV}$ (gas phase) and $-1.62 \mathrm{eV}$ (aqueous media) for the CP/Al-BN complex, $-1.9 \mathrm{eV}$ (gas phase), and - $1.47 \mathrm{eV}$ (aqueous medium) for the CP/Ga-BN complex, respectively. The electronic properties such as the HOMO-LUMO energy gaps decrease significantly by $48.28 \%$ and $47.95 \%$ in the gas phase and $42.27 \%$ and $38.41 \%$ in the aqueous medium after CP adsorption on Al-BN and Ga-BN, respectively. Quantum molecular descriptors predict that Al-BN and Ga-BN exhibit high sensitivity and reactivity of the PBN Nano sheet [6].

Also, M Cuevas-Flores et al. investigated the physical adsorption of cisplatin (CP) on graphene oxide (GO) and the reduction of graphene oxide ( $\mathrm{rGO}$ ) at the level of DFT theory by exploiting appropriate molecular models representing the most likely adsorption regions in GO and rGO nanostructures. . The results showed that the CP binding energy was enhanced concerning the interaction with native graphene. This is due to the preferential adsorption of the drug in the correspondence of the epoxy and hydroxy groups present on the GO core level: an energy decomposition analysis of the corresponding binding energy revealed that the most attractive contribution comes from the electrostatic attraction between the NH3 CP ends and the oxygen groups in (r) GO, which could be associated with the effects of hydrogen bonding. Moreover, it was found that the reaction of physically absorbed CP practically does not change. The free energy difference in the first hydrolysis reaction is almost identical to that of its free counterpart (unabsorbed drug). The reported results indicate that CP physical adsorption on GO and rGO carriers is possible as an exothermic process in an aqueous solution. The adsorption of CP can facilitate its solubility and transport in water solutions, exploiting the high tolerance of the peripheral carboxyl groups located at the edge of the GO and rGO nanostructures. Moreover, the higher affinity of CP concerning oxidative sites indicates a possible dependence of drug loading and release on $\mathrm{pH}$ conditions, which significantly facilitates its specific delivery [7].

However, to the best of our knowledge, recent experimental and theoretical work was devoted to different nanocage materials especially the GaN as potential drug carriers. At the same time, the GaN nanocage has only been synthesized [8], and no experimental or theoretical calculations are available to elucidate valuable information regarding its drug delivery ability. The present paper investigates Cisplatin molecules' adsorption and release processes on Ga ${ }_{12} \mathrm{~N}_{12}$ nanocage (GaN) with DFT calculations. Three orientations were designed to mimic the Cisplatin adsorbed on GaN. The adsorption energies and thermodynamic parameters were evaluated and compared for the eleven different adsorption systems. To investigate the interaction mechanism between the two components, the electronic properties such as the energies and orbitals distribution of the HOMOs, LUMOs, and their energy gaps (Eg), thermodynamic calculations. 


\section{Material and methods}

The simulations were executed using the density functional theory (DFT) depending on B3LYP (d,p) basis set which was performed in Gaussian 09 software package [9]. The B3LYP levels basis set depending on lanl2dz procedure were chosen according to its accuracy in electronic and structural properties especially for nanomaterials [10, 11]. The vibrational frequencies were also analyzed to inspect the true global lowest level of the predicted GaN nanocage.

Then, the cisplatin drug was adsorbed on these three nanoparticles and the chemical properties including charge transfer analysis, adsorption energies, dipole moment, energy gap, and recovery time were calculated to estimate the interaction between the drug molecule to nanoparticles.

To prophesy the chemical structural stability of the adsorbents and the adsorption energy was calculated using the equations below: [11-14]. One of the quantum chemical descriptors of hardness ( $\eta$ ) is defined as the half of the energy gap between highest occupied molecular orbital (HOMO) and the lowest unoccupied molecular orbital (LUMO) that:

$\eta \cong \frac{1}{2}\left(E_{\text {LUMO }}-E_{\text {НОМО }}\right) \cong \frac{1}{2}(I-A)$

A and I are known as the affinity of electrons and the ionization potential of the compounds.

Softness (S) can be calculated from hardness $(\eta)$ and hard soft acid-base (HSAB) principle is:

$S=\frac{1}{\eta}$

Electronegativity was calculated from Еномо, Ецимо by executing the Equation:

$\chi=-\frac{1}{2}\left(E_{\text {номо }}+E_{L U M O}\right)$

To calculate the effect of cisplatin on the energies of Fermi levels and the work functions of the nanocage, Fermi levels energy were calculated by using the equation from electronegativity, Fermi energy can be estimated that:

$E_{f}=E_{\mathrm{HOMO}}+\frac{\left(E_{L U M O}-E_{H O M O}\right)}{2}$

Several calculations were executed to calculate the total energies of the molecules depending on the position of the nanocage attached to the drug molecule. The adsorption energies of drug on the surfaces of the nanocage were obtained by:

$E_{\text {ads }}=E_{\text {complex }}-\left(E_{\text {nano }}+E_{\text {drug }}\right)$

Where $E_{\text {complex, }} E_{n a n o}$ and $E_{d r u g}$ donate the energy of complex composed with nano -drug and isolated energies of nanocage and drug, respectively. The Eads energy was determined from the summation of interaction energy (Eint) and deformation energies $\left(E_{\text {def }}\right)$ of drug (Edef-drug) and nanocage $\left(E_{\text {def-nano }}\right)$ during the adsorption process.

$E_{\text {int }}=E_{\text {complex }}-\left(E_{\text {nano in complex }}+E_{\text {drug in complex }}\right)$

$E_{\text {def }}=\left(E_{\text {drug in complex }}-E_{\text {drug }}\right)+\left(E_{\text {nano in complex }}-E_{\text {nano }}\right)$

Where $E_{\text {nano in complex, }} E_{\text {drug in complex }}$ are the energies of nanocage and drug with their geometries in the complex, respectively.

The thermodynamical parameters were also investigated like entropy $(\Delta S)$, the change of Gibbs free energy $(\Delta G)$, and enthalpy $(\Delta \mathrm{H})$, to examine the structural stability by the following equations was used [15]:

$\Delta G=G_{\text {complex }}-G_{\text {nanotube }}-G_{\text {drug }}$

$\Delta H=H_{\text {complex }}-H_{\text {nanotube }}-H_{\text {drug }}$ 
$\Delta S=\frac{\Delta H-\Delta G}{T}$

Where $\mathrm{H}_{\text {complex }}$ and $\mathrm{G}_{\text {complex }}$ are enthalpy and Gibbs free energy of drug adsorbed onto nanocage, $\mathrm{G}_{\text {nano }}$ and $\mathrm{H}_{\text {nano }}$ are the Gibbs free energy and enthalpy of the nanocage and $\mathrm{H}_{\text {drug }}$ and $\mathrm{G}_{\mathrm{drug}}$ are the enthalpy and Gibbs free energy of the drug respectively, $\mathrm{T}$ is the room temperature which is equal to $298.15 \mathrm{~K}$.

\section{Results and discussion}

The optimized geometries of pure GaN nanocage (Fig1 (a)) show that each nanocage comprises four and six-membered rings. The geometric parameters for optimized geometries in this study agree with the already reported data [16]. The geometric parameters of these pure nanocages have already been described in the literature; therefore, a detailed description is not provided here. We restrict our description of structures to those geometric parameters which are not well discussed previously. It is worth mentioning that both rings (four and six-membered) are not entirely planar.

Upon interaction with the drug, significant changes in the geometric parameters of these nanocages are observed. The interaction of the drug with this nanocage can be described in terms of binding energy and changes in geometric parameters. A list of important geometric parameters along binding energies is given in Table 2 . The binding energies of the drug GaN nanocage the energy values were $(-38.58,-28.77$, and -41.85$) \mathrm{Kcal} / \mathrm{mol}$ for the active atoms in the drug molecule (Pt, $\mathrm{N}$, and $\mathrm{Cl}$ ) respectively.

Moreover, the binding distance of the drug with the nanocage was equal to (2.4) $\AA$ before the adsorption. However, the interaction distances are different after adsorption. In our study, the distance had a noticeable change between the nanocage and the frug molecule. The high binding energies are indicative of the chemisorption of the drug on this nanocage.

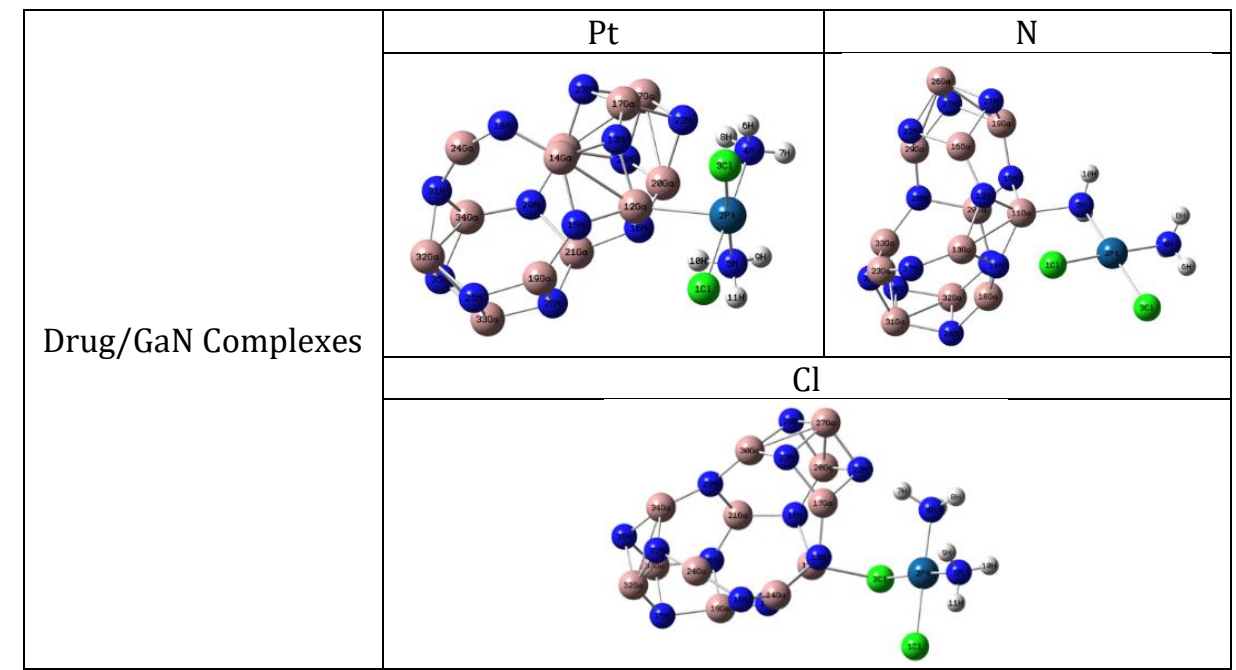

Figure 2 the optimized structure of the drug-complexes of GaN nanocage in all investigated locations

The recovery time (adsorption time) is one of the critical parameters used for both gas sensors and drug delivery systems, which predicts the amount of time required for drug adsorption from the adsorbent, as it is highly related to the adsorption energy, and it is known that a high adsorption reaction needs a high adsorption time and vice versa.

The recovery time is calculated by the equation: [17]

$\tau=\frac{1}{v} \operatorname{Exp}\left(\frac{-E_{a d s}}{k T}\right)$

Where $\mathrm{T}, \mathrm{k}$, and $v$ are the temperature, Boltzmann's constant and the attempted frequency, respectively. (K $2 \times 10-3$ $\mathrm{kcal} / \mathrm{mol} \mathrm{K}$ ) 
As the drug molecule seems to be adsorbed on the nanocages with maximum adsorption energy, a different recovery time (Table1) was obtained in vacuum UV- light conditions with frequencies of $3 \times 1016$ sec- 1 at room temperature.

Table1 The parameters of relaxed structures of isolated $\mathrm{X}_{12} \mathrm{Y}_{12}(\mathrm{X}=\mathrm{Al}, \mathrm{Y}=\mathrm{P})$ along with their complexes with the drug, the bond distance of the new complexes in $\AA$, recovery time (sec), and the adsorption energy (Kcal/mol)

\begin{tabular}{|l|c|c|c|}
\hline Structure & Distance $(\AA)$ After adsorption & Recovery time (sec.) & Eads (Kcal/mol) \\
\hline The drug(Pt)/GaN & 2.60 & $1.65 \times 10^{-12}$ & -38.58 \\
\hline The drug(N)/GaN & 1.95 & $2.52 \times 10^{-05}$ & -28.77 \\
\hline The drug(Cl)/GaN & 2.43 & $6.64 \times 10^{-15}$ & -41.85 \\
\hline
\end{tabular}

The results showed in Table 1 that the most significant value of the adsorption energy was at the bonding of the nanocage $(\mathrm{GaN})$ with chloride atom $\mathrm{Cl}$ with a value of $(-41.85) \mathrm{Kcal} / \mathrm{mol}$, which makes them suitable compounds to be used as drug delivery vehicles. However, the recovery time values were relatively considerable for all new complexes.

Table 2 Bond length between active atoms of the nanocage before and after adsorption of the drug in gas phases

\begin{tabular}{|l|c|c|c|}
\hline Structure & Bond location & Bond before adsorption & Bond after adsorption \\
\hline \multirow{3}{*}{ GAN/Drug(Pt) } & Ga-N16 & 1.85 & 1.96 \\
\cline { 2 - 4 } & Ga-N15 & 1.88 & 1.90 \\
\cline { 2 - 4 } & Ga-N13 & 1.87 & 1.92 \\
\hline \multirow{3}{*}{ GAN/Drug(N) } & Ga-N16 & 1.85 & 1.89 \\
\cline { 2 - 4 } & Ga-N15 & 1.88 & 1.99 \\
\cline { 2 - 4 } & Ga-N13 & 1.87 & 1.90 \\
\hline \multirow{3}{*}{ GAN/Drug(Cl) } & Ga-N16 & 1.85 & 1.90 \\
\cline { 2 - 4 } & Ga-N15 & 1.88 & 1.90 \\
\cline { 2 - 4 } & Ga-N13 & 1.87 & 1.93 \\
\hline
\end{tabular}

\subsection{Electronic properties and the relative stabilities}

To better understand the adsorption process of the drug on the GaN nanocage, frontier molecular orbitals (HOMO and LUMO) are analyzed before and after adsorption (Table 3).

The frontier molecular orbitals are given in Fig3. Energies of HOMO and LUMO of GaN nanocage are (-5.63 and -3.96) $\mathrm{eV}$ with a Fermi level of (-4.79) eV. The bandgap in GaN is (1.67) eV. The HOMO in GaN nanocage is mostly located on one side of the nanocage whereas the LUMO has density uniformly distributed on Gallium atoms.

Upon interaction with the drug, significant changes are observed in HOMO and LUMO. In the drug/GaN complexes upon interaction with the drug, energies of HOMO and LUMO are decreased to $(-5.86,-5.95$, and -5.59$)$ eV and $(-3.95,-3.89$, and -3.75$)$ eV respectively for the active atoms of the drug compound ( $\mathrm{Pt}, \mathrm{N}$, and $\mathrm{Cl}$ ), whereas the bandgap is increased $(1.91,2.06$, and 1.84)eV. (see Fig4 for details).

In order to understand the sensing mechanism of the nanocages to the drug, the percentage variation of $\% \Delta$ Eg energy gap during the adsorption process is taken into account by the following equation:

$\% \Delta E_{g}=100 x\left(E_{g 2}-E_{g 1}\right) / E_{g 1}$

Where $E_{g 1}$ and $E_{g 2}$ are the $E_{g}$ values of nanocage and complex structure, respectively. The percentage change values, of $\% \Delta E_{g}$ for each structure are given in Table 2 . 
Table 3 the orbital parameters: HOMO energies (EHOMO), LUMO energies (ELUMO), the energy of Fermi level (Ef), HOMO/LUMO energy gap (Eg) for the different new complexes

\begin{tabular}{|l|c|c|l|l|c|}
\hline System & EHOMO (eV) & ELUMO (eV) & Eg & $\Delta$ Eg & $\mathbf{E}_{\mathbf{f}}(\mathbf{e V})$ \\
\hline GaN & -5.63 & -3.96 & 1.67 & & -4.79 \\
\hline Drug(Pt)/GaN & -5.86 & -3.95 & 1.91 & 14.10 & -4.90 \\
\hline Drug(N)/GaN & -5.95 & -3.89 & 2.06 & 23.43 & -4.92 \\
\hline Drug $(\mathrm{Cl}) / \mathrm{GaN}$ & -5.59 & -3.75 & 1.84 & 10.22 & -4.67 \\
\hline
\end{tabular}

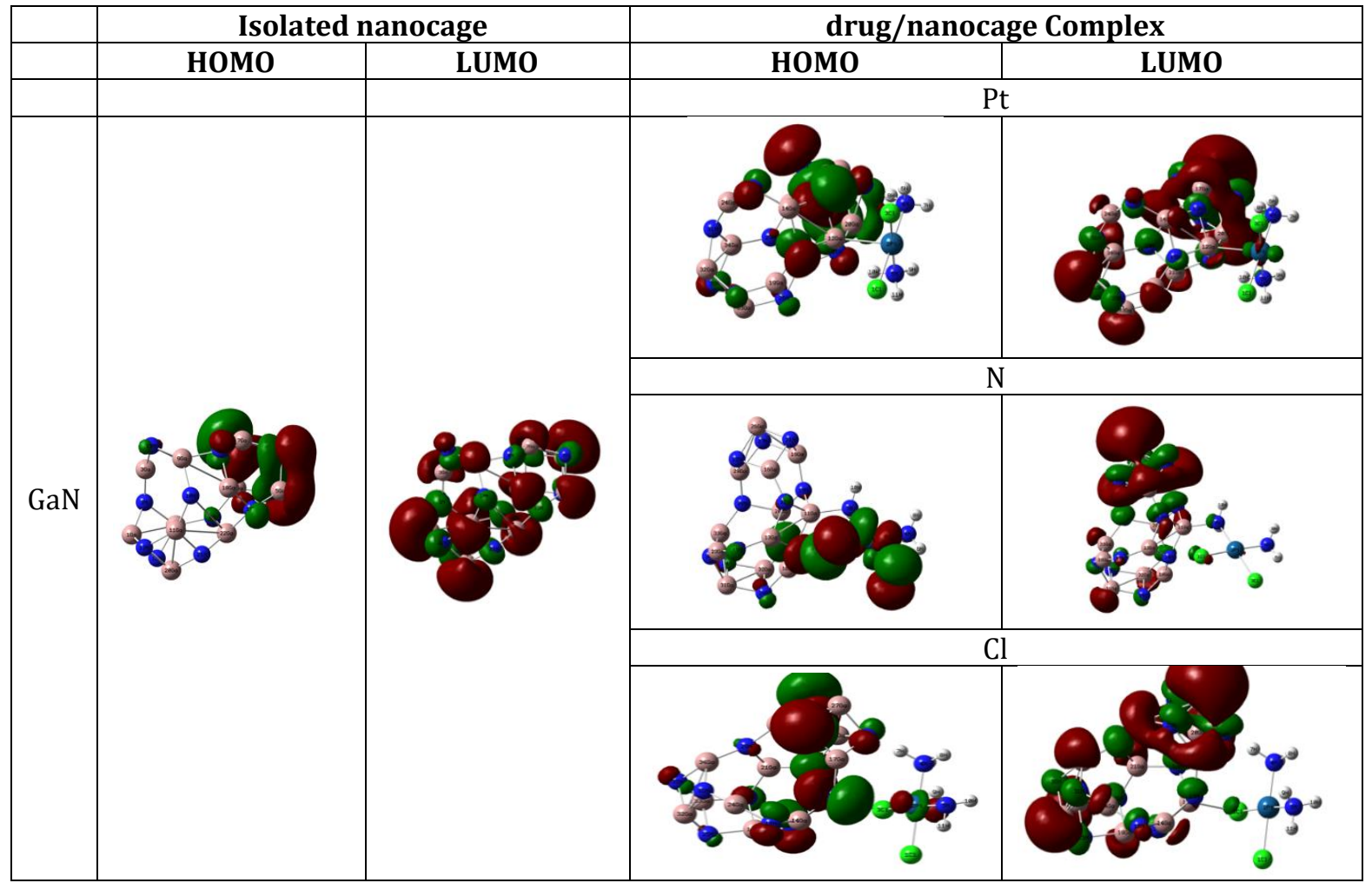

Figure 3 The HOMO-LUMO distributions of isolated nanocage along with their complex with the drug

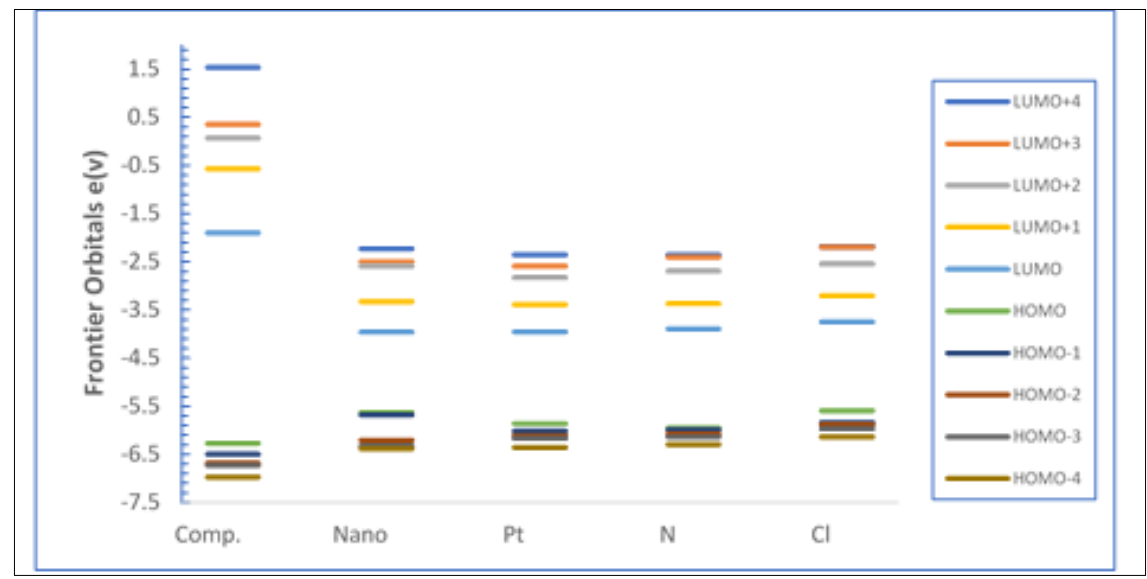

Figure 4 Energy gap between HOMO and LUMO for the drug, GaN nanocage, with all investigated locations regarding to the position of the nanocage on the drug 


\subsection{Quantum molecular descriptors}

The global indices of reactivity for the drug, GaN nanocage, and the drug adsorbed nanocage are given in Table 4.

The global reactivity indices are quite important parameters because they illustrate the reactivity and stability of a molecule or complex. The drug is relatively harder $(2.18 \mathrm{eV})$ compared to the nanocage studied. Interestingly, the hardness for the nanocage is in average on complexation. For GaN nanocage, the change in hardness $(\mathrm{y}=0.95,1.03$, and $0.92 \mathrm{eV}$ ) for all active atoms in the drug molecule $(\mathrm{Pt}, \mathrm{N}$, and $\mathrm{Cl}$ ) respectively.

Since hardness measures the stability of a chemical system towards deformation in the presence of an electrical field; therefore, the drug nanocage complexes are more prone to changes under electrical fields than pristine nanocages. Softness changes reciprocal to the hardness. Consequently, it is expected that softness for these complexes will increase. Changes in softness on adsorption of the drug, the nanocages are given in Table 3.

Table 4 Chemical potential $(\mu)$, hardness ( $\eta)$, softness $(S)$, and electrophilicity $(\omega)$ of the relaxed structure of BMSFBENZ adsorbed on GaN nanocage

\begin{tabular}{|l|c|c|c|c|}
\hline Structure & Chemical potential $(\boldsymbol{\mu})$ & hardness $(\mathbf{\eta})$ & softness $(\mathbf{S})$ & electrophilicity $(\boldsymbol{\omega})$ \\
\hline The drug & -4.08 & 2.18 & 0.45 & 3.82 \\
\hline $\mathrm{GaN}$ & -4.79 & 0.83 & 1.19 & 13.74 \\
\hline $\mathrm{Pt}$ & -4.90 & 0.95 & 1.04 & 12.61 \\
\hline $\mathrm{N}$ & -4.92 & 1.03 & 0.96 & 11.73 \\
\hline $\mathrm{Cl}$ & -4.67 & 0.92 & 1.08 & 11.83 \\
\hline
\end{tabular}

The potential chemical increases for all nanocages on complexation with the drug. The chemical potentials of GaN increase from (-4.08) eV. The greatest change in chemical potential is observed for $\mathrm{GaN}$ at the $\mathrm{Cl}$ atom. A relatively different trend is observed for electrophilicity. For the GaN nanocage, there was a noticeable increase except in the Pt atom (Table 4).

It is known that positive values of $\Delta \mathrm{H}$ and $\mathrm{G}$ indicate that the reaction process is endothermic rather than spontaneous. In contrast, negative values of $\Delta \mathrm{H}$ and $\mathrm{G}$ show that the reaction is an exothermic and spontaneous process [18] (see Table 5).

Table 5 Gibbs free energy $(\Delta \mathrm{G})$ in (kcal.mol-1), change of enthalpy $(\Delta \mathrm{H})$ in (kcal.mol-1), and change of entropy $(\Delta \mathrm{S})$ in (kcal/mol.K), minimum and maximum frequency in $\mathrm{cm}-1$ for the different complexes

\begin{tabular}{|l|l|l|l|l|l|}
\hline Structure & $\boldsymbol{\Delta G}$ & $\boldsymbol{\Delta} \mathbf{H}$ & $\boldsymbol{\Delta} \mathbf{S}$ & $\mathbf{V}_{\min }$ & $\mathbf{V}_{\max }$ \\
\hline $\mathrm{Pt} / \mathrm{GaN}$ & -39.40 & -53.81 & -0.04 & 26.74 & 3566.61 \\
\hline $\mathrm{N} / \mathrm{GaN}$ & -80.44 & -94.58 & -0.04 & 16.45 & 3580.54 \\
\hline $\mathrm{Cl} / \mathrm{GaN}$ & -46.24 & -60.06 & -0.04 & 17.90 & 3584.26 \\
\hline
\end{tabular}

\section{Conclusion}

In this study, the adsorption of the cisplatin $\mathrm{Cl}_{2} \mathrm{H}_{6} \mathrm{~N}_{2} \mathrm{Pt}$ drug on the GaN nanocage surface as a carrier was investigated at the B3LYP/lanl2dz (d,p) level of theory. Three stable systems are considered to find the most favorable adsorption structure/s of cisplatin/GaN Nanocage. These systems were identified as minimal points on the PES with no imaginary frequencies. The adsorption and release processes of the drug were considered. The adsorption energies corrected from BSSE of all investigated complexes are negative, indicating that the interactions are exothermic processes. The values of EBSSE ad for the three adsorbed systems are relatively large, indicating from a theoretical point of view a probable chemisorption process and the privilege of GaN as a carrier for the cisplatin drug. The configuration change indicates a significant difference between the two sets of systems. 


\section{Compliance with ethical standards}

\section{Authors' contributions}

All authors contributed to the study's conception and design. Material preparation, data collection, and analysis were performed by [Zaid Husham Al-Sawaff], [Fatma Kandemirli], and [Merve Şenğül ALPATER].

The first draft of the manuscript was written by [Merve Şenğül ALPATER], [Zaid Husham Al-Sawaff] under the supervision of [Fatma Kandemirli], and all authors commented on previous versions of the manuscript. All authors read and approved the final manuscript.

\section{Availability of data and material}

All the required data and calculations included in this research are original and not extracted from previous research, except for some paragraphs in the Introduction section taken as citations.

\section{Disclosure of conflict of interest}

The authors declare that they have no conflict of interests.

\section{References}

[1] Aldossary, S. A. Review on pharmacology of cisplatin: Clinical use, toxicity and mechanism of resistance of cisplatin. Biomedical and Pharmacology Journal, (2019). 12(1), 7-15. https://doi.org/10.13005/bpj/1608.

[2] Patra, J. K., Das, G., Fraceto, L. F., Campos, E. V. R., Rodriguez-Torres, M. D. P., Acosta-Torres, L. S., ... Shin, H. S. Nano based drug delivery systems: Recent developments and future prospects 10 Technology 1007 Nanotechnology 03 Chemical Sciences 0306 Physical Chemistry (incl. Structural) 03 Chemical Sciences 0303 Macromolecular and Materials Chemistry 11 Medical and He. Journal of Nanobiotechnology, (2018). 16(1), 1-33. https://doi.org/10.1186/s12951-018-0392-8.

[3] Zerenturk, A., \& Berber, S. Stability of GaN nanocages. Epl, (2013). 103(1). https://doi.org/10.1209/02955075/103/16003.

[4] Javan, M. B., Soltani, A., Azmoodeh, Z., Abdolahi, N., \& Gholami, N. A DFT study on the interaction between 5fluorouracil and B12N12 nanocluster. RSC Advances, (2016). 6(106), 104513-104521. https://doi.org/10.1039/c6ra18196a.

[5] Badiee, M. S., Morsali, A., \& Beyramabadi, S. A. DFT study on the adsorption of drug cisplatin onto carbon nanotubes. Oriental Journal of Chemistry, (2015). 31(4), 2053-2057. https://doi.org/10.13005/ojc/310425.

[6] Piya, A. A., Shamim, S. U. D., Uddin, M. N., Munny, K. N., Alam, A., Hossain, M. K., \& Ahmed, F. Adsorption behavior of cisplatin anticancer drug on the pristine, Al- and Ga-doped BN nanosheets: A comparative DFT study. $\begin{array}{lllll}\text { Computational and Theoretical Chemistry, } 1200 \quad \text { (2021,March), } & 113241 .\end{array}$ https://doi.org/10.1016/j.comptc.2021.113241.

[7] Cuevas-Flores, M. D. R., Bartolomei, M., García-Revilla, M. A., \& Coletti, C. Interaction and reactivity of cisplatin physisorbed on graphene oxide nano-prototypes. Nanomaterials, (2020). 10(6), 1-17. https://doi.org/10.3390/nano10061074.

[8] Zhu, Y. C., Bando, Y., Yin, L. W., \& Golberg, D. Hollow boron nitride (BN) nanocages and BN-nanocage-encapsulated nanocrystals. Chemistry - A European Journal, (2004). 10(15), 3667-3672. https://doi.org/10.1002/chem.200400002.

[9] D.J.F. M. J. Frisch, G. W. Trucks, H. B. Schlegel, G. E. Scuseria, M. A. Robb, J. R. Cheeseman, G. Scalmani, V. Barone, B. Mennucci, G. A. Petersson, H. Nakatsuji, M. Caricato, X. Li, H. P. Hratchian, A. F. Izmaylov, J. Bloino, G. Zheng, J. L. Sonnenberg, M. Had, G. Zheng, J. L. Sonnenberg, M. Hada, M. Ehara, K. Toyota, R. Fukuda, J. Hasegawa, M. Ishida, T. Nakajima, Y. Honda, O. Kitao, H. Nakai, T. Vreven, J.A. Montgomery Jr., J.E. Peralta, F. Ogliaro, M. Bearpark, J.J. Heyd, E. Brothers, K.N. Kudin, V.N. Staroverov, R. Kobayashi, J. Normand, K. Raghavachari, A. Rendell, J.C. Burant, S. S. Iyengar, J. Tomasi, M. Cossi, N. Rega, J.M. Millam, M. Klene, J.E. Knox, J.B. Cross, V. Bakken, C. Adamo, J. Jaramillo, R. Gomperts, R.E. Stratmann, O. Yazyev, A.J. Austin, R. Cammi, C. Pomelli, J.W. Ochterski, R.L. Martin, K. Morokuma, V.G. Zakrzewski, G.A. Voth, P. Salvador, J.J. Dannenberg, S. Dapprich, A.D. Daniels, "O. Farkas, J.B. 
Foresman, J. V Ortiz, J. Cioslowski, D.J. Fox, Gaussian 09, Revision D.01, Gaussian Inc., Wallingford. (2013). D0I: https://10.1017/CB09781107415324.004.

[10] M.R. Hossain, M.M. Hasan, N.-E. Ashrafi, H. Rahman, M.S. Rahman, F. Ahmed, T. Ferdous, M.A. Hossain, Adsorption behaviour of metronidazole drug molecule on the surface of hydrogenated graphene, boron nitride and boron carbide nanosheets in gaseous and aqueous medium: A comparative DFT and QTAIM insight, Phys. E LowDimensional Syst. Nanostruct. 126 (2021), 114483, https://doi.org/10.1016/j.physe.2020.114483.

[11] H. Rahman, M.R. Hossain, T. Ferdous, The recent advancement of low-dimensional nanostructured materials for drug delivery and drug sensing application: A brief review, J. Mol. Liq. 320-A (2020), 114427, https://doi.org/10.1016/j.molliq.2020.114427.

[12] extrinsic fullerenes as work function type sensors for amphetamine drug detection: DFT studies. Vacuum, (2016). 136, 156-162. https://doi.org/10.1016/j.vacuum.2016.12.003.

[13] Al-Sawaff, Z. ; Sayıner, H ; Kandemirli, F. Quantum chemical study on two benzimidazole derivatives 1,2.(2020). Retrieved from https://dergipark.org.tr/en/pub/jauist/issue/55760/739466.

[14] Kim, T. Il, Ri, C. Il, Yun, H. S., An, R. N., Han, G. B., Chae, S. Il, ... Jon, Y. A Novel Method for Calculation of Molecular Energies and Charge Distributions by Thermodynamic Formalization. Scientific Reports, (2019). 9(1), 1-12. https://doi.org/10.1038/s41598-019-56312-2.

[15] Al-Sawaff, Z., Dalgic, S., \& Kandemirli, F. Theoretical study of the adsorption of BMSF-BENZ drug for osteoporosis disease treatment on Al-doped carbon nanotubes (Al-CNT) as a drug delivery vehicle. European Journal of Chemistry, (2021). 12(3), 314-322. doi:10.5155/eurjchem.12.3.314-322.2143.

[16] Beheshtian, J., Bagheri, Z., Kamfiroozi, M., \& Ahmadi, A. A comparative study on the B 12N 12, Al 12N 12, B 12P 12 and Al 12P 12 fullerene-like cages. Journal of Molecular Modeling, (2012). 18(6), 2653-2658. https://doi.org/10.1007/s00894-011-1286-y.

[17] Sayiner, H. S., Kandemirli F., Senturk Dalgic S., Monajjemi M., Mollaamin F. Carbazochrome Carbon Nanotube as Drug Delivery Nanocarrier for Anti-bleeding Drug : Quantum Chemical Study, J. Molec. Modelling, (2021). 0-19.

[18] Chen, L., Feng, W., Fan, J., Zhang, K., \& Gu, Z. Removal of silver nanoparticles in aqueous solution by activated sludge: Mechanism and characteristics. Science of the Total Environment, (2020). 711, 135155. https://doi.org/10.1016/j.scitotenv.2019.135155 